\title{
Modulating p56Lck in T-Cells by a Chimeric Peptide Comprising Two Functionally Different Motifs of Tip from Herpesvirus saimiri
}

\author{
Jean-Paul Vernot, ${ }^{1,2}$ Ana María Perdomo-Arciniegas, ${ }^{1}$ \\ Luis Alberto Pérez-Quintero, ${ }^{1}$ and Diego Fernando Martínez ${ }^{1}$ \\ ${ }^{1}$ Fisiología Celular y Molecular, Facultad de Medicina, Universidad Nacional de Colombia, Bogotá 11001, Colombia \\ ${ }^{2}$ Instituto de Investigaciones Biomédicas, Facultad de Medicina, Universidad Nacional de Colombia, Bogotá 11001, Colombia
}

Correspondence should be addressed to Jean-Paul Vernot; jpvernoth@unal.edu.co

Received 17 March 2015; Revised 28 April 2015; Accepted 27 May 2015

Academic Editor: Darren R. Flower

Copyright (C) 2015 Jean-Paul Vernot et al. This is an open access article distributed under the Creative Commons Attribution License, which permits unrestricted use, distribution, and reproduction in any medium, provided the original work is properly cited.

\begin{abstract}
The Lck interacting protein Tip of Herpesvirus saimiri is responsible for T-cell transformation both in vitro and in vivo. Here we designed the chimeric peptide hTip-CSKH, comprising the Lck specific interacting motif CSKH of Tip and its hydrophobic transmembrane sequence (hTip), the latter as a vector targeting lipid rafts. We found that hTip-CSKH can induce a fivefold increase in proliferation of human and Aotus sp. T-cells. Costimulation with PMA did not enhance this proliferation rate, suggesting that hTip-CSKH is sufficient and independent of further PKC stimulation. We also found that human Lck phosphorylation was increased earlier after stimulation when T-cells were incubated previously with hTip-CSKH, supporting a strong signalling and proliferative effect of the chimeric peptide. Additionally, Lck downstream signalling was evident with hTip-CSKH but not with control peptides. Importantly, hTip-CSKH could be identified in heavy lipid rafts membrane fractions, a compartment where important T-cell signalling molecules (LAT, Ras, and Lck) are present during T-cell activation. Interestingly, hTip-CSKH was inhibitory to Jurkat cells, in total agreement with the different signalling pathways and activation requirements of this leukemic cell line. These results provide the basis for the development of new compounds capable of modulating therapeutic targets present in lipid rafts.
\end{abstract}

\section{Introduction}

Selective phosphorylation of tyrosine residues in the Tcell receptor- (TCR-) associated CD3 complex and $\zeta$ chains follows TCR engagement and activation by the MHC-peptide complex [1]. Tyrosine phosphorylation is mediated by protein members of the nonreceptor Src tyrosine kinase family, mainly p56Lck (Lck) and p59Fyn (Fyn) [2]. Lck is lymphoidspecific and essential for T-cell development and function $[3,4]$; it associates with surface molecules such as CD2, CD4, CD8, CD45, and IL-2 receptor [4-6]. Lck can be either phosphorylated by serine-threonine or tyrosine kinases but it is well known that its activity is mainly positively and negatively regulated by tyrosine phosphorylation in positions 394 and 505, respectively $[7,8]$. Stimulation of T-cell lines defective in Lck expression has shown an abnormal tyrosine phosphorylation pattern of downstream protein targets [9]. Lck has acquired importance as a therapeutic target for regulating T-cell response due to this central role in T-cell function [10-12].

Lck, as well as other Src family members, is also the target of viral proteins as a strategy for lymphotropic viruses [13], such as human immunodeficiency virus [14], Epstein-Barr virus [15], or Herpesvirus saimiri (HVS), to escape immune control and maintain latency [16]. In particular, HVS is a lymphotropic $\gamma$-herpesvirus, which is nonpathogenic in its natural host Saimiri sciureus, but in some New World primate species induces fulminant T-cell lymphomas [17]. HVS infection has been used in vitro in human and non-human primates (Aotus spp.) as a strategy for T-cell transformation 
[18-20]. This would suggest that transformation mechanisms in human and Aotus T-cells could have the same molecular basis.

Two HVS gene products, Tip (tyrosine kinase interacting protein) and StpC (Saimiri transforming protein), seem to be essential for the observed oncogenic phenotype [21]. Tip was able to induce T-cell lymphoma in transgenic mice and is therefore very likely to be responsible for the oncogenicity in T-cells [22]. A mechanistic model in which Tip participates in TCR signalling and CD4 downregulation has been proposed to explain HVS-infected T-cell longevity with consequences on viral persistence and pathogenesis [23]. Some studies have shown that Tip specifically associates with Lck, increasing its phosphorylation and activity and thus T-cell proliferation [24-26]. Moreover, Tip has been extensively implicated in oncogenic transformation $[13,27]$.

Nevertheless, in Jurkat T-cell lines or primary T-cells immortalized by lentiviral transduction, other authors have shown that Tip induces downregulation of Lck and an overall decrease in cellular tyrosine phosphorylation of several proteins, including Lck and ZAP70 [16, 28]. Early work has shown differences in signalling molecule expression and altered requirements for Jurkat cells activation [29], explaining in part the opposite effect of Tip in primary T-cells versus Jurkat cells or immortalized T-cells.

The Lck-binding domains (LBD) of Tip have been mapped to the carboxyl terminal portion of the molecule involving two independent binding motifs, $\mathrm{SH} 3 \mathrm{~B}$ ( $\mathrm{SH} 3$ binding) and CSKH (C-terminal Src kinase homology). They are included within a highly conserved region between amino acids 146 to 182 [30-33]. The SH3B motif (residues 172 to 182) binds to the SH3 domain on Lck, while the CSKH motif (residues 146 to 155) binds to the kinase catalytic domain [24]. Interestingly, mutants of Tip, containing either only SH3B or only CSKH domains, bind to Lck although to a reduced extent [32]. Also a truncated form of Tip lacking SH3B is still able to induce lymphomas in vivo [34] and coexpression of Lck with a Tip mutant lacking SH3B stimulated tyrosine phosphorylation of cellular proteins [32]. This suggests a suitable and sufficient role of the CSKH domain for Lck binding, T-cell signalling, proliferation, and eventually cell transformation.

As it would be expected for a protein regulating Lck, Tip has been shown to be constitutively present in lipid rafts [23, 35], a signalling platform for T-cell activation [36, 37], where early signals are induced allowing subsequently specific gene expression and proliferation $[26,38,39]$. The carboxyl terminal hydrophobic (hTip) sequence of Tip is responsible for its localization to lipid rafts [40]. In the present work, we have explored the intriguing possibility of inducing $\mathrm{T}$ cell activation and proliferation by using a short Lck binding motif of Tip (CSKH) properly delivered to lipid rafts. In fact, the hTip-CSKH chimera was delivered to detergent resistant membranes allowing us to specifically target Lck, to induce intracellular signalling and T-cell proliferation. Of great relevance, this work suggests that using a lipid raft targeting sequence from the transforming Tip protein could be a novel strategy to activate or inhibit signal transduction pathways, in other cell types and conditions, in which lipid raft dynamic is involved.

\section{Methods}

2.1. Peptide Synthesis and Characterization. Peptides were synthesized by solid phase as previously described [41]. The hTip sequence corresponds to the carboxyl-terminal residues 232-250 (CLVVVILAVLLLVTVLSIL); the CSKH motif EDLQSFLEKY plus a 6-residue extension (PPDFRK) adjacent to the CSKH motif was used as the hTip cargo, forming the chimera (CLVVVILAVLLLVTVLSILEDLQSFLEKYPPDFRK), here called hTip-CSKH. hTip, CSKH, and a chimeric peptide with the CSKH sequence in a scrambled configuration (hTip-CSKHsc) were also used in some experiments as controls. Peptides were analyzed by RP-HPLC and mass spectrometry to ascertain molecular weight and purity.

2.2. Blood Samples, Cell Isolation, and Cell Line. Human blood samples were obtained from healthy donors after informed consent. Aotus nancymaae from the Colombian Amazon region were housed according to NIH guidelines for animal handling. Both human and Aotus peripheral blood mononuclear cells (PBMC) were isolated by Ficoll-Hypaque density gradient centrifugation (Sigma-Aldrich Co., St. Louis, MO, USA). The Jurkat T-cell leukemia cell line (ATCC) was cultured under standard conditions. Cell viability was routinely assessed by Trypan-blue dye exclusion and only samples having $>90 \%$ viability were further used.

2.3. Aotus nancymaae Lck Sequence Determination. RNA from $1 \times 10^{7}$ lymph node cells was extracted by TRIZOL (Gibco, Invitrogen Corp., New York, NY, USA) as described elsewhere. Lck coding and proximal $5^{\prime}$ and $3^{\prime}$ UTR regions were amplified by RT/PCR using SuperScript III One Step RT/PCR with Platinum Taq (end point) (Invitrogen Life Technologies, Carlsbad, CA, USA) with sense primer LckRs17 (5'GCCTGGACCATGTGAAT3') and antisense primer LckRa20 (5'TGACTATGGCACAAGAAC$\left.\mathrm{TC}^{\prime}\right)$. Three clones were obtained and sequenced using 6 different primers: LckRs17, LckRa20, M13 forward, M13 reverse primers, and two internal primers LckIntF (5' TGGACAGTTCGGGGAGG3') and LckIntR02 (5'ATGATGTAGATGGGCTCCT3 ${ }^{\prime}$ ). Sequencing was performed with BigDye 3.1 sequence kit and analyzed in ABI PRISM 310 genetic analyzer, with the ABI PRISM Sequencing Analysis 3.3 MT Navigator 1.0.2. (Perkin Elmer, Foster City, CA, USA) and Chromas 1.45 software. Other sequencing was done by MACROGEN Inc. Overlapping sequences were aligned using ClustalX 1.83 software and analyzed by GeneDoc software (multiple sequence alignment editor, version 2.5.000). The sequence submitted to GenBank (accession number: AY821852) was the consensus sequence from the three clones. Human, Saimiri sciureus, and Aotus nancymaae (accession numbers: NP_005347, CAC38871, and AAV70114, resp.) protein sequences were compared by using the same software. Sequences used were found in the GenBank protein databases.

2.4. Peptide FITC-Labeling and Fluorescence Microscopy. hTip-CSKH and scrambled CSKH peptides were labeled with fluorescein isothiocyanate (FITC). Shortly, $4.75 \mathrm{~mL}$ of 
a $0.0125 \mathrm{mM}$ solution of peptide was incubated $24 \mathrm{~h}$ in $0.1 \mathrm{M}$ carbonate-bicarbonate buffer with $250 \mu \mathrm{L}$ of a $2.5 \mathrm{mM}$ FITC solution in DMSO. Dialysis was performed during $48 \mathrm{~h}$ against $0.1 \mathrm{M}$ ammonium chloride in $1 \%$ DMSO (membrane of exclusion limit of $3.5 \mathrm{kDa}$; Spectrum Laboratories Inc., Rancho Dominguez, CA, USA). $10^{5}$ human PBMC resuspended in RPMI 1640 supplemented with 10\% FCS, $10 \mathrm{mM}$ HEPES, and $1 \mathrm{mM}$ sodium pyruvate (complete medium) for $1 \mathrm{~h}$ at $37^{\circ} \mathrm{C}$ were incubated with $40 \mu \mathrm{M}$ of FITC-labeled hTip-CSKH or CSKH(Scr). Cells were washed in PBS and resuspended in $20 \mu \mathrm{L}$ of media ( $150 \mathrm{mM} \mathrm{NaCl}, 50 \%$ glycerol) and mounted in a glass microscope slide with a coverslip and observed in a Nikon C-1plus fluorescence microscope. Digital photographs were taken with a Sony DSC-P73 digital camera.

2.5. Lymphocyte Proliferation Assays. A total of $5 \times$ $10^{4} \mathrm{PBMC} /$ well were cultured for $1 \mathrm{~h}$ at $37^{\circ} \mathrm{C}$ and $5 \%$ $\mathrm{CO}_{2}$ in complete medium in the absence or presence of different peptide concentrations (as indicated in each figure) in 96-well flat-bottomed microplates (Linbro, Aliso Viejo, CA, USA). In some experiments, $\mathrm{PBMC}$ were also stimulated with $2 \mu \mathrm{g} / \mathrm{mL}$ PHA-P or $25 \mathrm{ng} / \mathrm{mL}$ PMA for $48 \mathrm{~h}$ or $72 \mathrm{~h}$ with or without addition of peptides. Afterwards, $0.5 \mu \mathrm{Ci} /$ well [methyl-3H]-thymidine (ICN Biomedicals, Inc., Irvine, CA, USA) was added to cultures for the last $18 \mathrm{~h}$. Cells were then harvested (PHD Harvester, Cambridge Tech) in glass fiber strips (Cambridge Technology, Watertown, MA, USA) and assayed for [methyl-3H]-thymidine incorporation by liquid scintillation counting $\beta$-scintillation system Beckman LS 6500 (Beckman Instruments, Fullertown, CA, USA).

Peptides-treated or peptides-untreated Jurkat cells $(5 \times$ $10^{4}$ ) were stimulated with $2 \mu \mathrm{g} / \mathrm{mL}$ ionomycin or $2 \mu \mathrm{g} / \mathrm{mL}$ PHA-P and processed as described above for primary cells. The stimulation index percentage (SIP) was defined as being the ratio of mean [methyl-3H]-thymidine (counts per minute) incorporated in the presence of a peptide to that incorporated in the absence of peptide $(\times 100)$.

2.6. Protein Tyrosine Kinase Immunostaining. PBMC from humans were preincubated (or not) for $1 \mathrm{~h}$ with $60 \mu \mathrm{M}$ hTipCSKH and further stimulated with PHA-P $(5 \mu \mathrm{g} / \mathrm{mL})$ for 15,30 or $60 \mathrm{~min}$. Stimulation was stopped by ice-cooling the cultures; these cells were then washed twice in ice-cold PBS and cell extracts were prepared by a 20-minute incubation in lysis buffer $(20 \mathrm{mM}$ Tris-Cl pH $8.0,276 \mathrm{mM} \mathrm{NaCl}$, $10 \%$ glycerol, $1 \%$ NP40, $1 \mathrm{mM}$ PMSF, $10 \mu \mathrm{g} / \mathrm{mL}$ aprotinin, $10 \mu \mathrm{g} / \mathrm{mL}$ leupeptin, $1 \mathrm{mM} \mathrm{Na} 3 \mathrm{VO} 4,10 \mathrm{mM} \mathrm{NaF}$, and $2 \mathrm{mM}$ EDTA). Extracts were centrifuged for $15 \mathrm{~min}$ at $10,000 \mathrm{~g}$ and $4^{\circ} \mathrm{C}$; supernatants were recovered and subjected to $10 \%$ SDSPAGE as described elsewhere. Proteins were then transferred to PVDF membranes (Immobilon-P, Millipore Corp., Bedford, MA, USA). Immunodetection was performed with anti-Lck (clone MOL171) antiphosphotyrosine (Clone PY20) (Pharmingen, San Diego, CA, USA). In other sets of experiments, human PBMC were stimulated with PHA-P or hTip$\mathrm{CSKH}$ and cell extracts prepared as described above. Proteins were then transferred to PVDF membranes and immunodetection was performed with anti-Fyn (AHO0482, Invitrogen) and anti- $\beta$-Actin (sc-47778, Santa Cruz Biotechnology). For data analysis, p56Fyn and p59Fyn bands were normalized to $\beta$-actin.

2.7. Downstream Signaling after hTip-CSKH Stimulation. For ERK 1/2 phosphorylation, human PBMC were stimulated for $2 \mathrm{~h}$ with the different peptides or with PHA-PMA as described above. Cells extracts were prepared as above and assayed by WB with anti-p-ERK1/2 (sc-7383, Santa Cruz Biotechnology) and anti-ERK1/2 (sc-7383, Santa Cruz Biotechnology). Erk phosphorylation was normalized to total ERK1/2.

2.8. Biochemical Isolation of Detergent Resistant Membranes (DRMs). $6.5 \times 10^{7}$ human PBMC were treated with $40 \mu \mathrm{M}$ of hTip-CSKH in serum-free RMPI-1640 during 1 hour at $37^{\circ} \mathrm{C}$. The cells were washed in medium and the membranes were extracted during $20 \mathrm{~min}$ in $200 \mu \mathrm{L}$ of ice-cold lysis buffer containing Tris $25 \mathrm{mM}$ pH 7.4, $150 \mathrm{mM} \mathrm{NaCl}$, $2 \mathrm{mM}$ EDTA, $0.5 \%$ Triton-X100, $1 \mathrm{ug} / \mathrm{mL}$ leupeptin, $1 \mathrm{ug} / \mathrm{mL}$ pepstatin, $4 \mathrm{ug} / \mathrm{mL}$ aprotinin, $1 \mathrm{mM}$ PMSF, $1 \mathrm{mM} \mathrm{Na} 3 \mathrm{VO} 4$, and $50 \mathrm{mM} \mathrm{NaF}$. For sucrose density gradients, cell extracts were mixed with $250 \mu \mathrm{L}$ of $80 \%$ sucrose in TNE buffer (Tris $25 \mathrm{mM} \mathrm{pH} \mathrm{7.4,} 150 \mathrm{mM} \mathrm{NaCl}$, and $2 \mathrm{mM}$ EDTA) and overlayed with $730 \mu \mathrm{L}$ of $35 \%$ sucrose and $320 \mu \mathrm{L} 5 \%$ sucrose and centrifuged at $200,000 \mathrm{~g}$ during $5 \mathrm{~h}, 4^{\circ} \mathrm{C}$ (Beckman Optima, rotor TLS-55). Eight fractions of equal volume were recovered from the top of the gradient, diluted with $1 \mathrm{~mL}$ of TNE, and detergent resistant membranes in each fraction were precipitated by ultracentrifugation at $100,000 \mathrm{~g}$ during $45 \mathrm{~min}, 4^{\circ} \mathrm{C}$. The pellets were extracted in $20 \mu \mathrm{L}$ of Laemmli buffer: half used for WB and Flotillin-2 detection and the other half for SDS-PAGE in Tris-Tricine buffer for peptide identification as described [42]. To facilitate the identification of the peptides in this system, erythrocyte membranes were prepared in parallel and incubated with the different peptides used. Peptides were identified by sample separation in TrisTricine SDS-PAGE.

\subsection{PBMC Surface Marker Characterization after hTip-CSKH} Treatment. PBMC were incubated for $24 \mathrm{~h}$ in the absence or presence of $60 \mu \mathrm{M}$ hTip-CSKH peptide, PBS washed, suspended in $0.5 \%$ BSA-PBS solution, and further incubated for 20 min at RT with the respective monoclonal antibody. AntiCD3 PE-conjugated antibody (clone UCTH1) was purchased from Sigma (Sigma-Aldrich Co., St. Louis, MO, USA); and anti-TCR $\alpha / \beta$ FITC-conjugated antibodies were purchased from Pharmingen (San Diego, CA, USA). All incubations with fluorochrome-tagged antibodies were done in the dark. Then, cells were washed with $2 \mathrm{~mL}$ of PBS and centrifuged. After a final wash, cells were suspended in $0.5 \mathrm{~mL}$ PBS and immediately read in a FACScan flow cytometer (Beckton Dickinson, BD Biosciences, San Jose, CA, USA).

2.10. Statistical Analysis. Paired statistical analyses were performed using Student two-tailed $t$-test. Paired two-tailed $t$ test analyses were performed for the data comparing values obtained from nontreated and hTip-CSKH or hTip-treated cells at the same time points. 


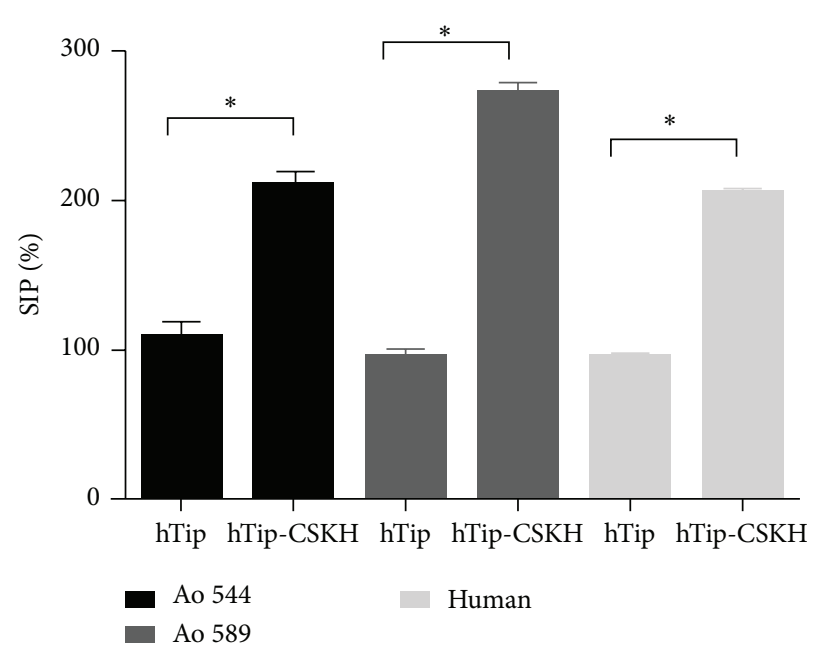

(a)

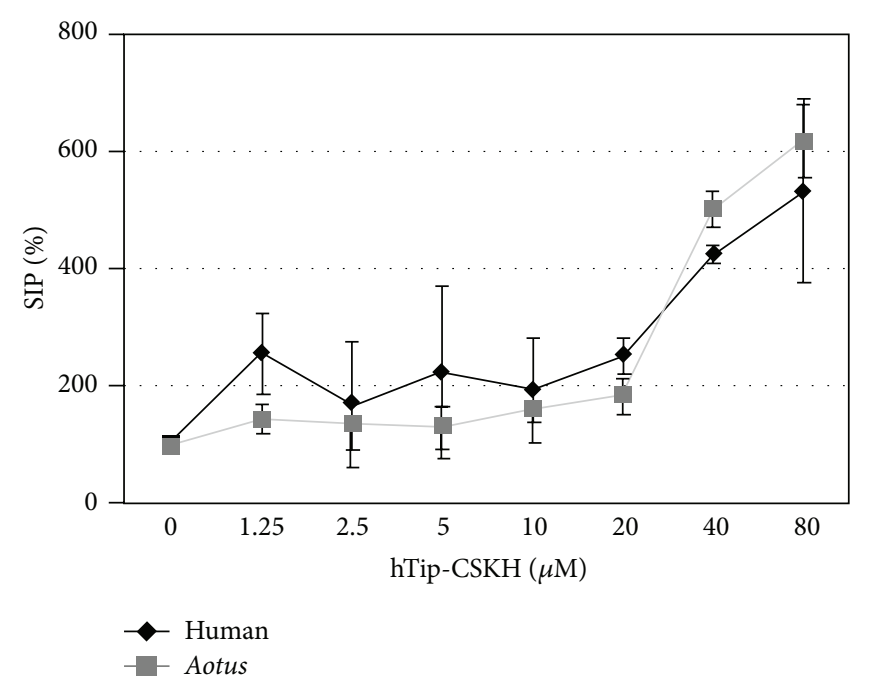

(b)

FIgURE 1: hTip-CSKH induced cell proliferation in human and Aotus cells. (a) PBMC from two Aotus nancymaae and one human donor were treated with $40 \mu \mathrm{M}$ hTip or hTip-CSKH peptides and assayed for [3H]-thymidine incorporation after 48 -hour treatment. (b) Human and Aotus PBMC exposed to several hTip-CSKH concentrations were assessed for [3H]-thymidine incorporation after 72 -hour treatment. Stimulation index percentage (SIP) is shown (see Section 2). Student's $t$-test statistically significant values $(p<0.05)$ are marked with asterisk $(*)$.

\section{Results}

3.1. High Identity in Lck and Similarity in T-Cell Proliferation in Response to hTip-CSKH between Aotus and Humans. The Aotus primate experimental model has proved to be useful for basic immunology and vaccination studies. The establishment of similarities is essential for physiological studies involving immune modulation, although some differences in $\mathrm{T}$-cell response have been found [43]. Previously, we found that Aotus Lck was more related to Saimiri sciureus than to human Lck. Nevertheless, high identity (>98\%) was also observed between human and Aotus Lck protein sequences (Supplementary Figure 1, see Supplementary Material available online at http://dx.doi.org/10.1155/2015/395371). Functionally important sequences for molecular docking (i.e., CENCH motif for CD4/CD 8 coreceptors binding and $\mathrm{SH} 2$ phosphotyrosine binding domain) or catalysis (ATP binding region, 364D and $273 \mathrm{~K}$ residues) were highly conserved. Differences between human and Aotus Lck sequences rely only on 12 residues.

Cell proliferation in Aotus and humans has different requirements: while Aotus PBMC respond poorly to PHA$\mathrm{P}$, they readily respond to favin stimulation [43]. On the other hand, it is well known that human lymphocytes require at least two different signals (signals 1 and 2) for proliferation [44]. These signals can be simulated in vitro by the combination of calcium influx (signal 1) and PKC activation (signal 2) or PHA-P stimulation (both signals). Interestingly, incubation of Aotus or human PBMC with hTip-CSKH $(40 \mu \mathrm{M})$ for $48 \mathrm{~h}$ induced increased proliferation (2-3 times) (Figure 1(a)). The increased proliferation was dependent on the cargo sequence since proliferation of PBMC treated with hTip alone or the chimeric CSKH scrambled peptide
(hTip-CSKHsc) was as low as that of control cells without peptide (not shown). Some variability in Aotus response to hTip-CSKH compared to humans was observed, but this is probably due to what we have previously described for this experimental model [43]. The hTip-CSKH effect is then similar to PHA-P, that is, inducing proliferation by its own, although to a lesser extent. The result observed with hTipCSKH was stronger and dose-dependent in both human and Aotus PBMC when proliferation was assayed after 72hour incubation and with different peptides concentrations (Figure 1(b)). Increased proliferation was initially observed between 20 and $40 \mu \mathrm{M}$ but, remarkably, a sixfold proliferation induction was observed at $80 \mu \mathrm{M}$ hTip-CSKH and 72-hour incubation in both human and Aotus T-cells without losing cell viability.

3.2. hTip-CSKH Induces Proliferation in Human T-Cells Independently of Signal 2. As indicated, proliferation induced by the polyclonal T-cell activator PHA-P in Aotus PBMC is lower compared to humans. Also human PBMC proliferation induced by hTip-CSKH was lower (40\% or more dependent on the conditions) than that usually induced by PHA-P, suggesting different mechanisms of induction and/or different strength of activation. To address this issue initially, we examined hTip-CSKH proliferative effect on previously PHA-P-stimulated Aotus T-cells. In spite of the variability reported previously and observed above, there was a statistically significant increase in proliferation induced by hTipCSKH (Figure 2) in 5 Aotus PBMC samples that have been previously activated by PHA-P. As expected, this increase was low (11-22\%) but statistically significant. Control hTip peptide treatment did not increase proliferation, confirming 


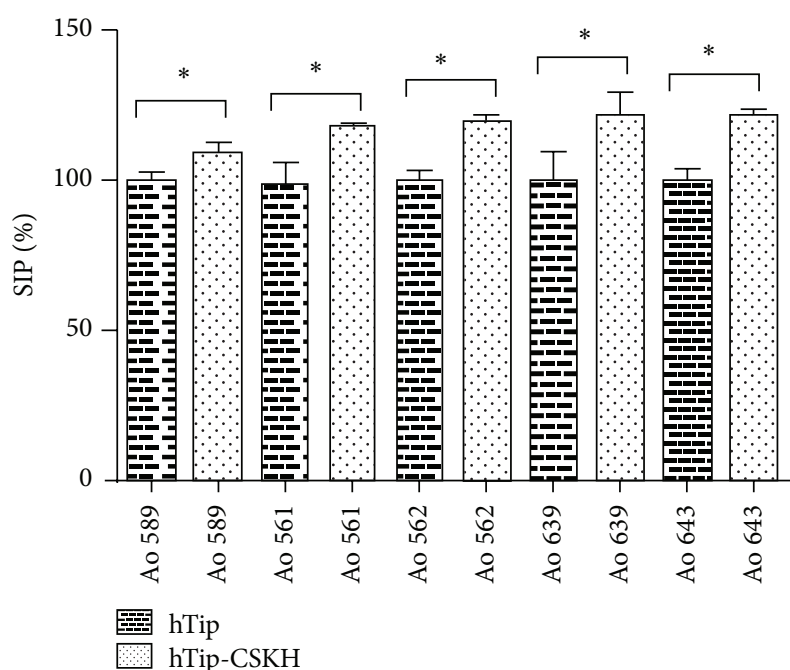

FIGURE 2: hTip-CSKH even induced a slight increase in proliferation in PHA-P-stimulated Aotus nancymaae PBMC. Five Aotus nancymaae PBMC samples were treated with hTip and hTip-CSKH peptides $(40 \mu \mathrm{M})$; after 1-hour incubation, $2 \mu \mathrm{g} / \mathrm{mL}$ PHA-P was added to the culture. $[3 \mathrm{H}]$-thymidine incorporation was assessed after $72 \mathrm{~h}$ of culture. Student's $t$-test statistically significant values $(p<0.05)$ are marked with asterisk $(*)$.

that the proliferative effect is therefore cargo specific. Our results show that T-cells that have been previously stimulated by the strong activator PHA-P can still be further activated by the chimeric peptide hTip-CSKH, suggesting either that stimulation with PHA-P is not maximal in this condition and hTip-CSKH delivers a synergic proliferation signal or that hTip-CSKH signals through other pathway(s).

To further explore this topic, we used the classical protein kinase C (PKC) activator phorbol-12-myristate-13-acetate (PMA) which induces signal 2 [44], bypassing cell surface receptors stimulation. We hypothesized that PKC activation will act as a complementary signal to hTip-CSKH. As expected, a relatively low cell proliferation was obtained when human PBMC were treated only with PMA. Addition of hTipCSKH induced a dose-dependent increase in proliferation (starting at $20-40 \mu \mathrm{M}$ ) with a maximum fivefold increase at $80 \mu \mathrm{M}$ when compared to PMA-treated cells; the control peptide hTip did not produce the same effect (Figure 3). The onset of cell proliferation and maximal response to hTip-CSKH was very similar to that obtained without PMA (compare Figures 1(b) and 3). Therefore, hTip-CSKH seems to be able to efficiently replace signal 2 on its own. Aotus PBMC were not used in these PMA experiments, since we have previously shown that under certain circumstances (and for unknown reasons) these cells can respond to either signal 1 or signal $2[43]$.

3.3. hTip-CSKH Effect on the T-Cell Line Jurkat. Jurkat T-cells were very interesting to further test hTip-CSKH effect, since TCR activation in this cell line causes growth inhibition, suggesting that these cells have different activation requirements [45]. Jurkat cells do not respond to PHA-P stimulation but

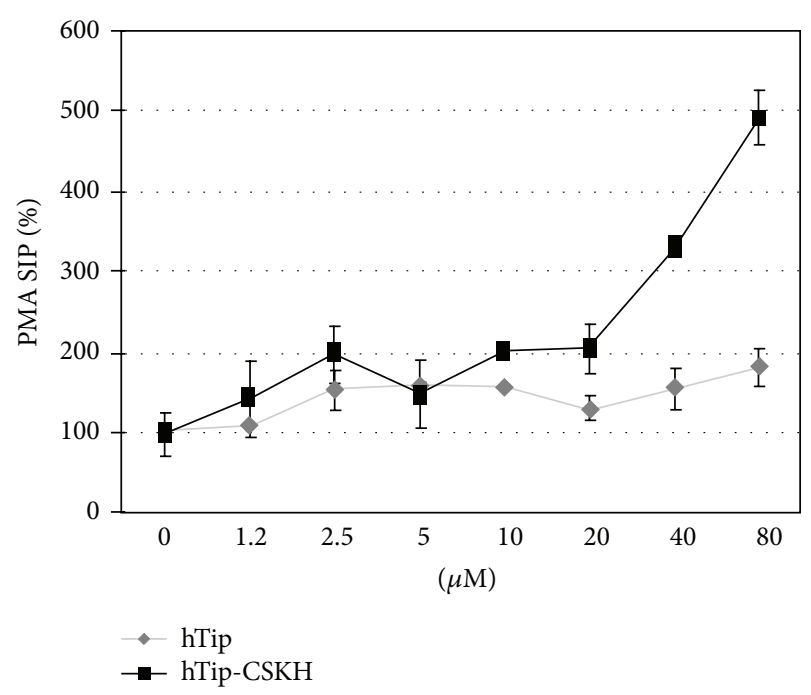

FIGURE 3: hTip-CSKH induced similar 5-fold increased proliferation in PMA-stimulated PBMC. Human PBMC exposed to different hTip-CSKH or hTip concentrations for $1 \mathrm{~h}$ were stimulated with $25 \mathrm{ng} / \mathrm{mL}$ PMA. Cells were assessed for [3H]-thymidine incorporation after $72 \mathrm{~h}$. Stimulation index percentage (SIP) is shown.

respond adequately to calcium influx. Figure 4(a) shows that Jurkat cells had a proliferation rate about two- to threefold higher in the presence of ionomycin than unstimulated cells. As it has been previously reported, PHA-P-stimulated cells showed a slight reduction in the proliferation rate (Figure 4(a)). Interestingly, hTip-CSKH treatment of Jurkat cells also showed a slight diminution in proliferation, similar to what we have seen before with PHA-P (compare Figures 4(a) and 4(b), 3rd column with NS controls). Control hTip peptide had no effect (Figure 4(b), 2nd column). Ionomycinstimulated Jurkat cell treated with hTip-CSKH showed a strong reduction in proliferation rate while control hTiptreated cells showed no effect (Figure 4(b)). Together, these results have shown that if T-cells are able to proliferate in response to PHA-P (signals 1 and 2 induction), as in normal human and Aotus T-cells, then h-Tip-CSKH will also induce proliferation. In contrast, if stimulation through signal 1 inhibits proliferation (as in Jurkat cells), then hTipCSKH will also be inhibitory. This would give support to our previous suggestion that signaling by hTip-CSKH peptide is strongly enough (and probably includes both signals) to induce proliferation on its own.

3.4. hTip-CSKH Can Be Identified in Detergent Resistant Membranes (DRMs). It has been previously reported that the transmembrane domain (amino acid residues 229-250) of Tip is required for its association with lipid rafts [35]. We then explored biochemically hTip-CSKH localization at the plasma membrane, specifically its association with DRM. Since DRMs localize into the low-density fractions in sucrose gradients, they have usually been associated with lipid rafts [37]. First, we tested an electrophoresis system (SDS-PAGE in Tris-Tricine buffer) having high-resolution 


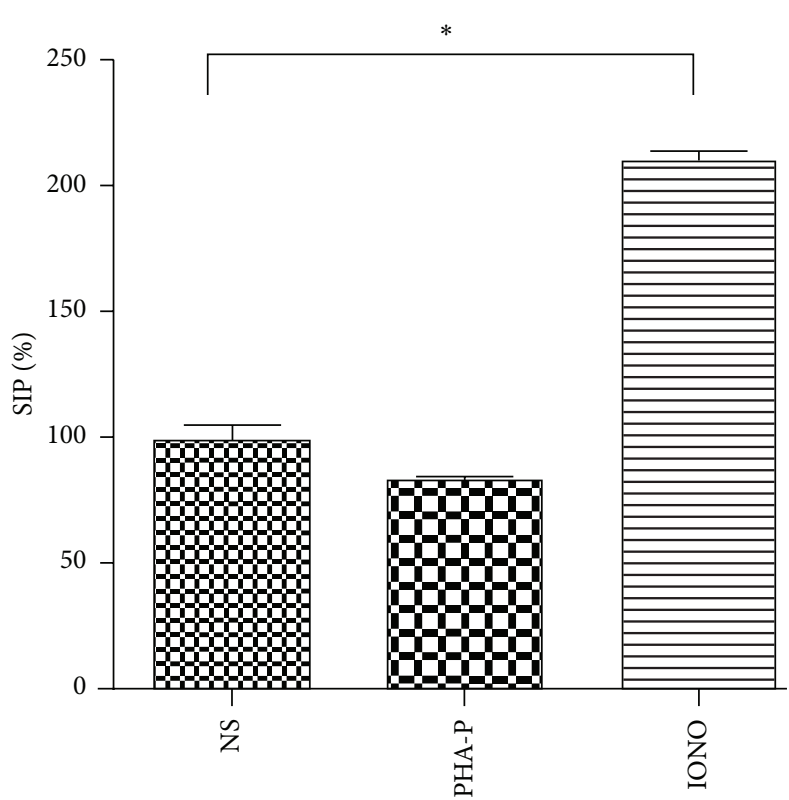

(a)

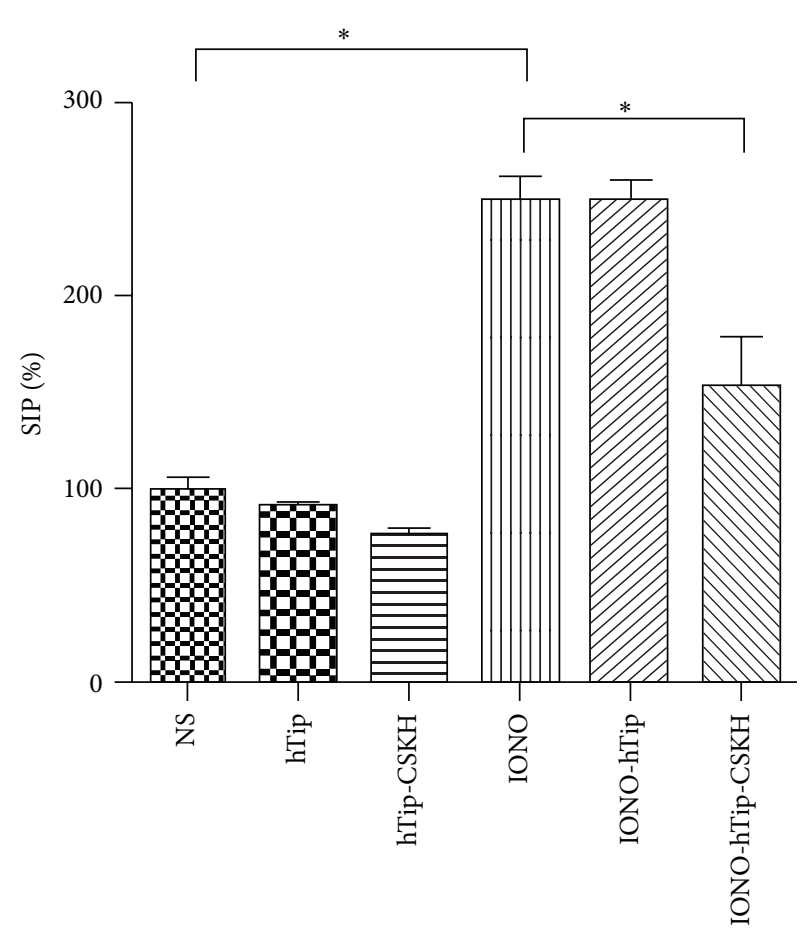

(b)

FIGURE 4: hTip-CSKH induced a decreased cell proliferation in Jurkat cells. (a) Jurkat cells were treated with PHA-P or ionomycin and assayed for $[3 \mathrm{H}]$-thymidine incorporation after 48 -hour treatment. (b) Jurkat cells were treated with hTip, hTip-CSKH for $1 \mathrm{~h}$, and then ionomycinstimulated and assayed for [3H]-thymidine incorporation. Jurkat cells treated with each peptide alone or nontreated peptides were added as controls. Stimulation index percentage (SIP) is shown (see Section 2). NS: nonstimulated; Iono: ionomycin. Student's $t$-test statistically significant values $(p<0.05)$ are marked with asterisk $(*)$.

power for peptides identification compared to classical SDSPAGE. $10 \mu \mathrm{g}$ of each of hTip-CSKH, hTip, hTip-CSKHsc, or CSKH peptides was mixed with $10 \mu \mathrm{L}$ of an erythrocyte cell membranes extract. As it can be seen in Figure 5(a), hTip-CSKH, hTip-CSKHsc, and CSKH could be identified as diffuse and fast migrating bands below the $6,9 \mathrm{kDa}$ MW standard (arrow). hTip could not be resolved probably because of its high hydrophobicity. We next prepare a Triton X100 extract of hTip-CSKH-treated PBMC and fractionated it by sucrose density gradient centrifugation. Eight fractions were collected and assayed for hTip-CSKH (by TrisTricine SDS-PAGE) and Flotillin-2 (by SDS-PAGE and WB) presence. Flotillin-2, a marker of lipid rafts, was distributed mainly in fractions 1-3 and 6-8 (Figure 5(b), lower panel). A band corresponding to the hTip-CSKH MW was detected in fractions 6 and 7 (Figure 5(b), upper panel, arrow), showing hTip-CSKH localization in membrane rafts. The existence of a novel type of membrane raft-like microdomains (heavy DRM), containing a number of membrane signaling molecules, including LAT and Lck, was recently demonstrated [46]. The above evidence suggests that almost all of the chimeric hTip-CSKH is present in these novel heavy rafts, where it could interact with the Lck protein. Additionally, incubation of human PBMC with the FITC-labeled hTip$\mathrm{CSKH}$ during $1 \mathrm{~h}$ and analysis by fluorescence microscopy showed a homogeneous surface peripheral localization and no intracytoplasmatic accumulation (Supplementary Figure 2). This shows that hTip-CSKH interacts with the cytoplasmic membrane and is localized in lipid rafts.

3.5. hTip-CSKH Targets Lck and Induces Its Early Activation. The heavy lipid rafts are part of a TCR signaling platform involved in the early coordination of T-cell signaling events, namely, the activation of nonreceptor protein tyrosine kinases [46]. We have analyzed the hTip-CSKH effect on the lymphocyte-specific protein tyrosine kinase Lck, key molecule during T-cell activation, and hypothetical target of the hTip-CSKH chimeric peptide used here. Antibodies directed against human Lck and phosphotyrosine residues were used for assessing human Lck phosphorylation during time-course experiments, following T-cell short stimulation with PHA-P in the presence or absence of hTip-CSKH. Cell extracts were prepared after $0,15,30$, and $60 \mathrm{~min}$ of stimulation; WB detection was simultaneously performed for Lck and phosphotyrosine residues. Defined bands of about $56 \mathrm{kDa}$ and $59 \mathrm{kDa}$ were evident in both blots (Figure 6(a)). When analyzing Lck staining at time point 0 (Figure 6(a), top panel), the p56Lck band appeared more intense than the p59Lck band in cells with PHA-P without hTip-CSKH. After 15 min of T-cell stimulation, a progressive increase in p59Lck band staining and tyrosine phosphorylation was noted and this lasted for the rest of the period tested (Figure 6, top left 


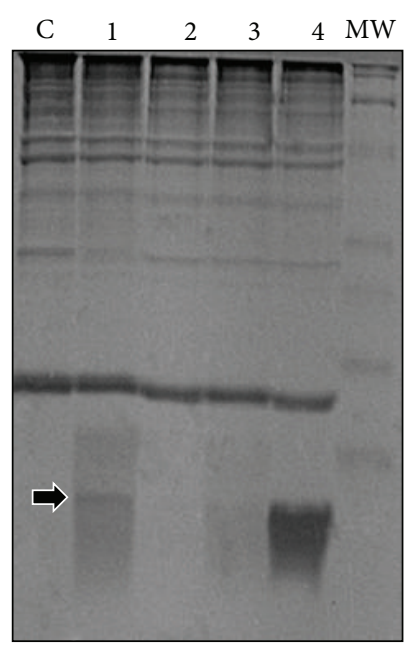

(a)

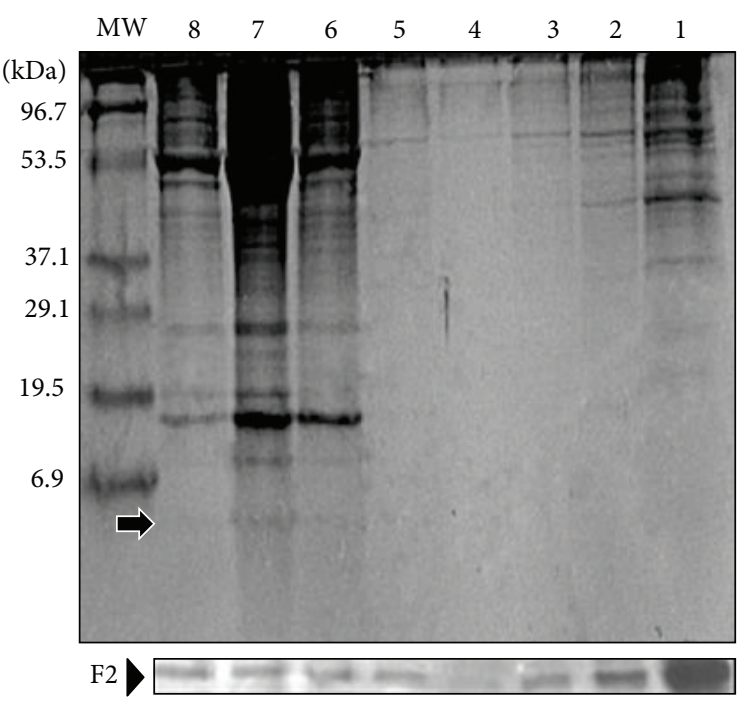

(b)

FIGURE 5: Tris-Tricine electrophoretic identification of hTip-CSKH or control peptides. (a) $10 \mu \mathrm{g}$ of the indicated peptides $(1=\mathrm{hTip}-\mathrm{CSKH}$; $2=$ hTip; $3=$ hTip-CSKH-Scr; $4=\mathrm{CSKH}$ ) was mixed with $10 \mu \mathrm{L}$ of a protein extract of red blood cells membranes. The gel was stained with Coomassie Brilliant Blue. The size of each molecular weight marker (MWM) is indicated in the right and the control (c) membranes without peptides in the left. The arrow indicates the band corresponding to hTip-CSKH molecular weight. (b) The membrane extract from hTip-CSKH-treated human PBMC was resolved by ultracentrifugation in a sucrose density gradient. Eight fractions were recovered as indicated in each line. Half of each fraction was used for Tris-Tricine electrophoresis for hTip-CSKH identification (the arrow marks the band corresponding to hTip-CSKH molecular weight). The remaining amount of the fraction was used for WB blot in order to know its Flotillin 2 content (bottom) and to differentiate between light (lanes 1-3) and heavy (lanes 5-8) fractions. The size of each molecular weight marker (MWM) is indicated in the left.

panel). This occurred simultaneously with reduced p56Lck band intensity, suggesting that a more intense p59Lck band depended on the contribution of p56Lck band during PHA$\mathrm{P}$ stimulation. On the contrary, in hTip-CSKH-treated cells, the ratio between p59Lck and p56Lck bands became inverted immediately after stimulation and lasted for the period tested (Figure 6). This rapid increase in Lck phosphorylation after hTip-CSKH and PHA-P stimulation is consistent with the observed increase in proliferation induced by hTip-CSKH in T-cells that have previously been activated with PHA$\mathrm{P}$ (Figure 2). As it would be expected, hTip-CSKH induced on its own Lck phosphorylation and increased tyrosine phosphorylation (Figure 6(c)) in full agreement with the proliferation experiments in Figure 1.

As it has been reported that Fyn is structurally related to Lck and has at least in part a redundant role during T-cell activation [47], we tested hTip-CSKH effect on this protein tyrosine kinase. Since Fyn activation depends on Lck activation [48] and the latter seems to be still maximal after $1 \mathrm{~h}$ of PHA-P or hTip-CSKH stimulation (Figure 6), we tested hTip-CSKH effect on Fyn after $2 \mathrm{~h}$ of peptide treatment. As it can be seen in Figure 7(a), PHA-P stimulation induces both the reduction in the intensity of the $59 \mathrm{kDa}$ Fyn protein (by about 22\%) and the appearance of a low MW band (56 kDa). hTip-CSKH had a similar effect (20\% reduction) on the $59 \mathrm{kDa}$ Fyn protein while the second band appeared diffused and poorly resolved. Thus, hTip-CSKH induces a different effect on Fyn when compared to PHA-P stimulation, suggesting a specificity towards Lck. Additionally, it can be concluded that the increase seen in the Lck band of $59 \mathrm{kDa}$ (Figure 6) is not due to an increase in the Fyn band since, on the contrary, hTip-CSKH induces a reduction in the Fyn $59 \mathrm{kDa}$ band. It might also be considered that although hTipCSKH induced early activation signals and later proliferation, the signal induced must, of course, be different from the PHA$\mathrm{P}$ signal and far from being complete.

3.6. Downstream Signaling Events Induced by hTip-CSKH. To further evaluate hTip-CSKH induced signal strength, we studied Erk phosphorylation, an important step in TCR-induced proliferation [49]. The ERK phosphorylation level achieved during human PBMC stimulation with both PHA-P and PMA was 5.5-fold when compared to control unstimulated cells (Supplementary Figure 3). hTip-CSKH produced 2.5-fold induction in ERK2 phosphorylation; the other peptides tested had minimal or no effect, as evaluated by densitometry. We observed that hTip peptide treatment induced a 1.5 -fold increase in ERK1/2 phosphorylation but we considered this effect to be not relevant as it was not enough to induce T-cell proliferation as it was shown above. We conclude then that hTip-CSKH was able to activate signals that are known to occur downstream of Lck.

3.7. hTip-CSKH Does Not Downregulate Cell Surface Markers. HVS infection induced a reduction in TCR, CD4, CD3, and $\mathrm{CD} 2$ expression. It was shown that this downregulation was 


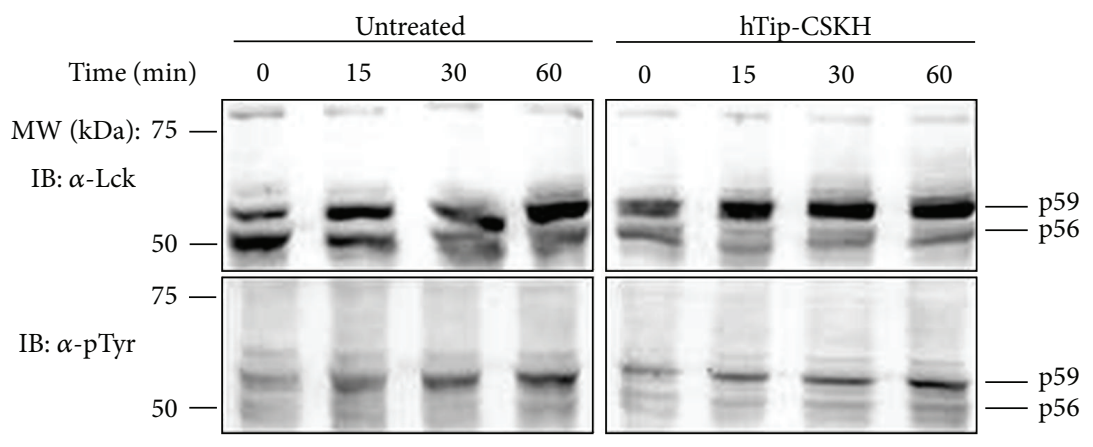

(a)

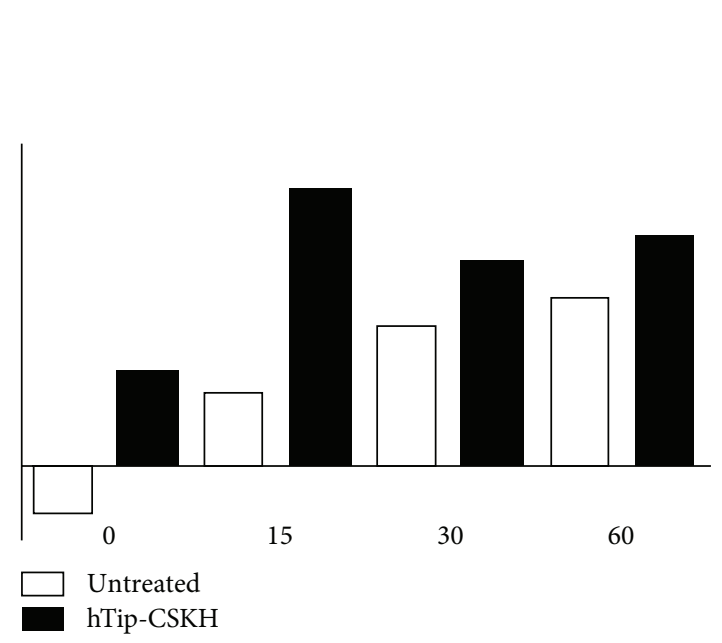

(b)

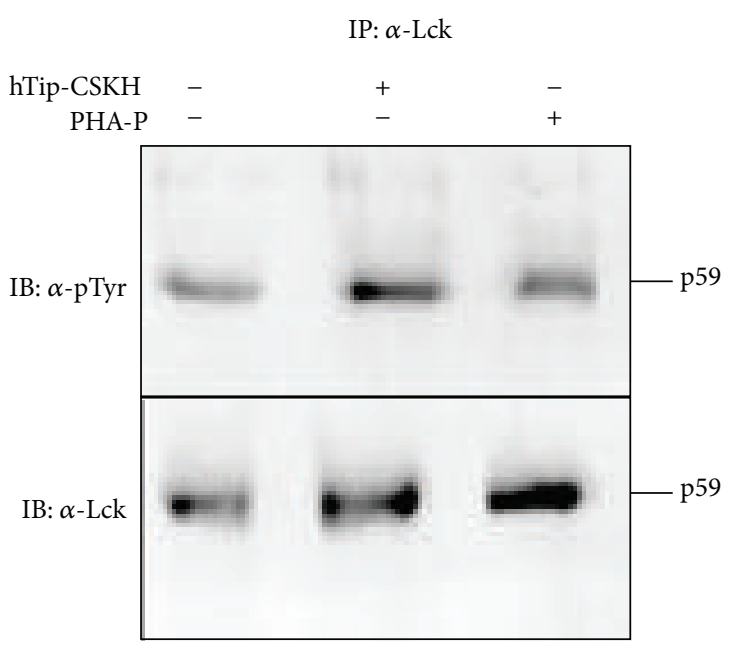

(c)

FIGURE 6: Time-course assay for Lck in human PBMC after cell stimulation. (a) PBMC either untreated (lanes 1-4) or treated (lanes 5-8) for $1 \mathrm{~h}$ with hTip-CSKH were stimulated with PHA-P for 0, 15, 30, and $60 \mathrm{~min}$ and then were lysed. Extracts were subjected to SDS-PAGE and proteins transferred to PVDF membranes. WB was performed with Lck (upper panels) and P-Y (lower panels) monoclonal antibodies. Two bands were clearly defined which correspond to the low molecular weight (p56) and the high molecular weight band (p59), respectively. (b) Quantification of p56 and p59 bands showed an increase of the p59/p56 ratio. White bars represent nontreated cells and black bars hTipCSKH-treated cells p59/p56 ratio at different time points. (c) Lck immunoprecipitation of PBMC extracts after stimulation with PHA-P or hTip-CSKH for $30 \mathrm{~min}$. WB was performed with monoclonal antibodies against pTyr and Lck as indicated.

partially dependent on Tip interactions with the cellular protein Tap (Tip-associated protein) and mapped to an aminoterminal portion of Tip, different from the Lck binding domain [50]. Therefore, 24-hour hTip-CSKH-treated human PBMC were evaluated for surface marker expression. We observed that CD3 and TCR expression was identical in hTipCSKH-treated and untreated cells (Supplementary Figure 4), suggesting that the effect of Tip on cell surface receptor downregulation and proliferation could be dissociated by using hTip-CSKH.

\section{Discussion}

HVS infection transforms both non-human primates and human T-cells to TCR-independent proliferation [18-20]. Tip binding to Lck and subsequent Lck activation are partially responsible for this effect $[24,32]$. We have previously performed the characterization of Lck in Aotus nancymaae and compared them to human Lck. The high identity (98\%) found when comparing both species suggests that Lck functionality in Aotus T-cells is very similar to humans and that synthetic peptides designed for human Lck binding and modulation could also do so in Aotus Lck.

The HVS Tip carboxyl-terminal hydrophobic (hTip) sequence was selected in this study as a cargo vehicle since it has been demonstrated that Tip is constitutively present in lipid rafts $[23,35]$ and there is experimental evidence connecting hTip with Tip localization in lipid rafts [40]. Additionally, Tip binds specifically to Lck through SH3B and CSKH motifs. Given that a Tip mutant without the SH3B domain was able to transform T-cells [21], we focused on the other Tip motif (CSKH) responsible for Lck binding and activity. It has previously been shown that both motifs can bind Lck independently [32]. Thus, a chimeric peptide formed by hTip and Tip's CSKH motif was synthesized to study its ability to modify T-cell physiology by targeting Lck.

Our results showed a clear increase (2-3 times) in thymidine incorporation in human and Aotus PBMC treated with the chimeric hTip-CSKH peptide for $48 \mathrm{~h}$. When longer stimulation periods were used (e.g., $72 \mathrm{~h}$ ), a 5- to 6-fold increase 


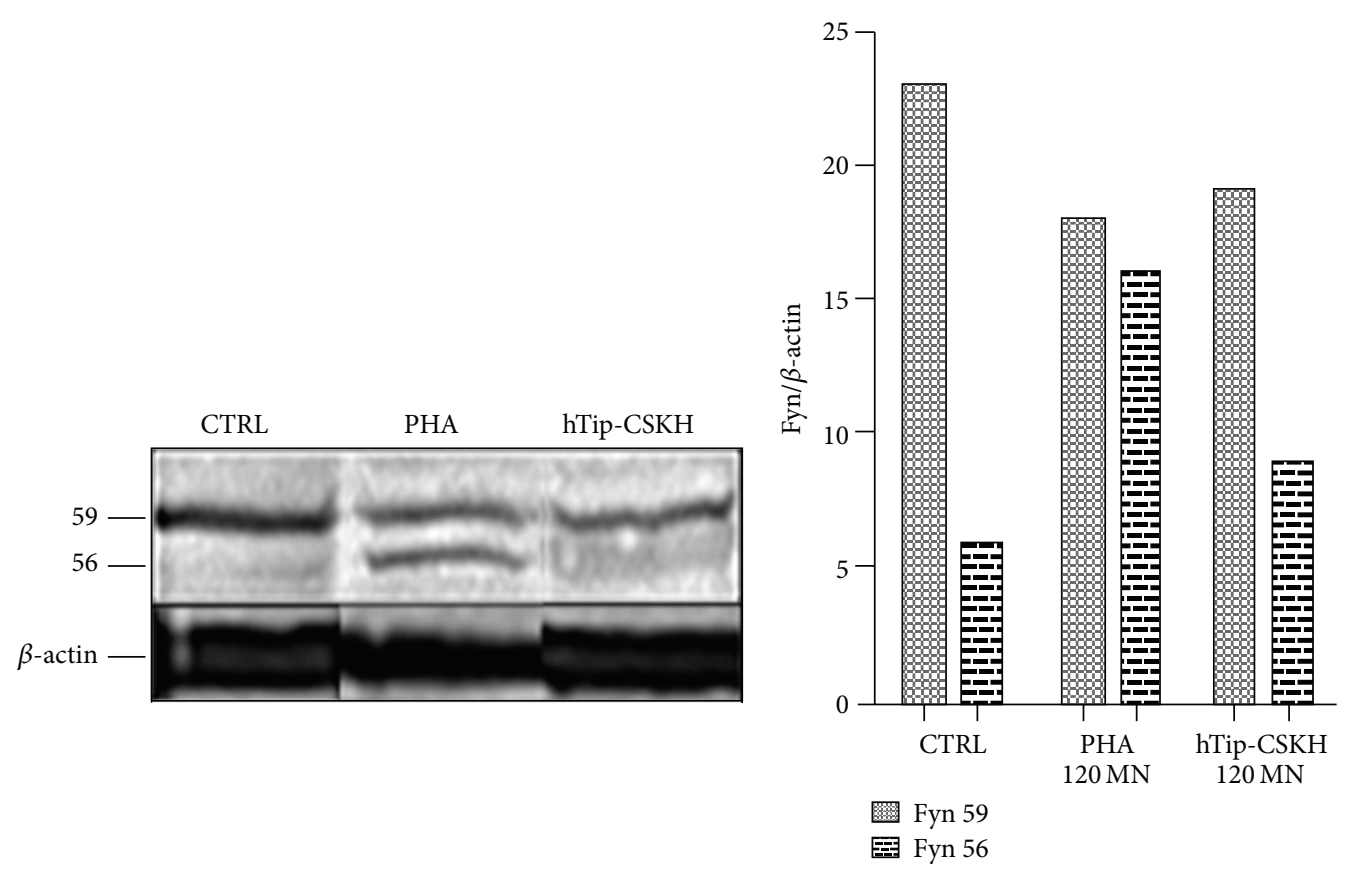

(a)

(b)

FIgURE 7: Fyn detection in stimulated human PBMC. (a) PBMC were treated with PHA-P and hTip-CSKH during $2 \mathrm{~h}$, lysed, and the protein extract resolved by SDS-PAGE. Western blot was performed with an anti-Fyn specific antibody. PHA-P was used as positive control, which induces the appearance of a second band of $56 \mathrm{kDa}$. (b) Quantification of the Fyn p59 and p56 bands. The intensity of each Fyn band was normalized to the loading control $\beta$-actin.

in proliferation was observed. Taking into consideration that PHA-P induces multiple receptors engagement (with a strong intracellular signaling input), the fact that hTip-CSKH by its own can increase proliferation is remarkable since hypothetically only one specific pathway is being targeted by the use of a unique Lck binding motif (CSKH). Interestingly, after Tcell PHA-P stimulation, hTip-CSKH could further increase proliferation (11-22\%), suggesting either a strong hTip-CSKH effect or additional hTip-CSKH stimulation through other mechanisms. This effect was cargo and sequence specific, since control peptides (hTip, hTip-CSKHsc, or CSKH) did not have any effect on proliferation.

Human T-cells require the simultaneous induction of at least two different signaling pathways for full activation, one through the TCR (signal 1) and the second through costimulatory molecules (signal 2). These signals can be mimicked by mitogenic stimulation (PHA-P) or the use of calcium ionophores (signal 1) plus PK-C activation (signal 2) [51]. hTip-CSKH-treated or hTip-treated cells were stimulated with the PK-C activator PMA to test whether signal 2 could further improve hTip-CSKH effect. A 5-fold increase in proliferation was observed with hTip-CSKH, suggesting either that the chimeric peptide is strong enough to induce both signals, as with PHA-P stimulation, or that, under very strong and atypical signal 1 stimulation, signal 2 is not anymore required.

Further evidence of this assumption was obtained by studying hTip-CSKH effect on the T-cell leukemia cell line
Jurkat. These cells do not proliferate in response to TCR activation by mitogens (PHA-P) or to anti-CD3 antibodies; even an opposite effect, driving cells to an apoptotic state, has been shown [45]. Tip expression in these cells has also been used as a model for Lck modulation [52]. We have also shown here that hTip-CSKH induces a slight decrease in proliferation, equivalent to PHA-P treatment. This inhibition was more evident and statistically significant when ionomycin-stimulated (signal 1) Jurkat cells were used. It is possible that an apoptosis process is taking place, as in the case of PHA-stimulated Jurkat cells [45]. Thus, if stimulation via the TCR signaling pathway causes cells to proliferate, then hTip-CSKH treatment has a proliferation inducing effect too. On the contrary, if the TCR signaling pathway induces an inhibitory proliferative effect, as what happens in Jurkat cells, hTip-CSKH will also cause this outcome. Although very similar responses to PHA-P stimulation were observed in primary (stimulation) or transformed T-cells (inhibitory) with hTip-CSKH, clearly, an atypical T-cell stimulation by hTip-CSKH will hardly match completely with a classical activation model.

After PBMC hTip-CSKH stimulation and DRM extraction, we could identify the chimeric peptide in fractions containing "heavy" lipid rafts having high content of LAT, Lck, and Ras signaling molecules [46]. Recently, it was shown that an amphipathic helical sequence of 14 amino acids, enriched in charged and hydrophobic amino acids and contiguous to the Tip TM sequence, could increase Tip localization to 
lipid rafts [40]. Our cargo CSKH sequence has about the same length of this sequence (16 aa) and is also enriched in charged and hydrophobic amino acids, suggesting a similar effect in lipid rafts localization. This membrane microdomain localization was essential to support our initial considerations regarding the use of this hTip sequence and its capacity to deliver a cargo sequence with the capacity to modulate Lck. In fact, we have shown here that hTip-CSKH induces a rapid increase in the appearance of the p59Lck band with a concomitant reduction in p56Lck. These changes are due to Lck phosphorylation and have been previously observed after TCR stimulation [53-55]. This effect was similar in PBMC stimulated only with PHA-P, although the activation kinetics in the presence of the chimeric peptide was extremely rapid. This suggests that hTip-CSKH induces a strong and rapid Lck activation and is responsible on its own for the induced proliferation observed. This is in agreement with the strong proliferation effect of hTip-CSKH shown above.

As Fyn can replace Lck, under certain circumstances, and there is a close sequence and structural identity between both tyrosine kinases, it was interesting to study hTip-CSKH effect on Fyn. Here, a reduction in the p59Fyn band was determined excluding its participation in the observed effect on Lck phosphorylation. The other changes observed in Fyn, especially the appearance of a diffuse p56Fyn band, deserve further experimentation. Nevertheless, these results are in agreement with those that have shown that Tip effect is specific for Lck, not for Fyn or Lyn [25], and consistent with their described differences in protein association and subcompartmental localization [48]. Interestingly, in the TCR signaling models, Fyn activation is dependent on Lck and this process is coordinated spatially in lipid rafts. How this could affect Fyn activation is not known. Since both kinases, Lck and Fyn, have been shown to play a role in the different models of T-cell anergy [56], it would be interesting to explore the hTip-CSKH capacity to revert this process in T-cell.

One of the protein kinases responsible for Lck phosphorylation in Ser residues is ERK, which was activated here too after hTip-CSKH stimulation. As shown before, Lck phosphorylation on Y394 and Y505 is crucial for modulating its activity [57]. Some authors have shown that, after activation, Lck autophosphorylates its Y394 residue further increasing its catalytic activity [58]. We therefore assessed Lck tyrosine phosphorylation and found that p59Lck was more phosphorylated than p56Lck. Taken together, our results have shown that hTip-CSKH induces enhanced T-cell proliferation by targeting and activating Lck. More biochemical and structural work in this field is needed to completely elucidate the mechanism of Lck activation by hTip-CSKH.

By using the hTip-CSKH peptide, the above-described effects on Lck and lymphocyte proliferation could be molecularly separated from the effects caused by other Tip domains. It has been shown that Tip amino-terminal region is particularly involved in cell surface molecules downregulation through Tip binding to a cytosolic p80 protein [23]. We have shown here that surface expression of CD3 and TCR $\alpha \beta$ was not altered after hTip-CSKH treatment. It was therefore possible to separate the effects induced by Tip CSKH motif from those obtained when the whole Tip molecule was used.

Targeting proteins involved in signal transduction, like protein tyrosine kinases $[11,59]$ or protein tyrosine phosphatases $[60,61]$, is considered to be a viable strategy for therapeutic intervention. The use of defined motifs for blocking or inducing a particular function in these signaling molecules is a valuable approach for the fine modulation of cellular processes. We have shown here that the information contained in discrete protein sequences (i.e., CSKH) could be sufficient to modulate complex biological responses, provided that they are duly delivered to specific subcellular compartments. To our knowledge, this is the first report that uses a chimeric peptide to modulate T-cell signaling in lipid rafts. This would undoubtedly be a valuable tool for therapeutic intervention to target molecules whose dynamics depends on lipid rafts.

\section{Conclusions}

We have developed a novel chimeric peptide to modulate Lck signaling in cell membrane lipid rafts. In fact, hTipCSKH induced on its own strong cell proliferation in normal lymphocytes as a consequence of the specific activation of the lipid rafts-anchored Lck. Interestingly, hTip-CSKH was inhibitory to Jurkat cells, in total agreement with the different signaling pathways and activation requirements of this leukemic cell line. We propose that hTip could be an effective vehicle for delivering a cargo sequence to lipid rafts, and its use could be extended for other molecules responsible for cancer cell growth.

\section{Disclosure}

The current address for Ana María Perdomo-Arciniegas is Colombian Cord Blood Bank, Hemocentro Distrital, District Secretary of Health, Bogotá, Colombia, and that for Luis Alberto Pérez-Quintero is Neuroimmunology Laboratory, Centre de Recherche du Centre Hospitalier de l'Universite de Montréal (CRCHUM), Montréal, QC, Canada.

\section{Conflict of Interests}

The authors declare that there is no conflict of interests regarding the publication of this paper.

\section{Acknowledgments}

The authors are especially grateful to Dr. Manuel E. Patarroyo for his support and to staff members of the FIDIC for Aotus blood samples; also to Mr. Jason Garry for grammatical correction of the paper and Jeremy Tabery in Nonlinear Dynamics Ltd. for the shareware version of TotalLab software. They also want to thank MACROGEN Inc. for DNA sequencing. This study was partially financed by the División de Investigación Bogotá (DIB) and the Faculty of Medicine to Jean-Paul Vernot, Universidad Nacional de Colombia. 


\section{References}

[1] E. N. Kersh, A. S. Shaw, and P. M. Allen, "Fidelity of T cell activation through multistep T cell receptor $\zeta$ phosphorylation," Science, vol. 281, no. 5376, pp. 572-575, 1998.

[2] T. Mustelin and K. Taskén, "Positive and negative regulation of T-cell activation through kinases and phosphatases," Biochemical Journal, vol. 371, no. 1, pp. 15-27, 2003.

[3] S. J. Anderson, S. D. Levin, and R. M. Perlmutter, "Involvement of the protein tyrosine kinase p56lck in T cell signaling and thymocyte development," Advances in Immunology, vol. 56, pp. 151-178, 1994.

[4] R. Zamoyska, A. Basson, A. Filby, G. Legname, M. Lovatt, and B. Seddon, "The influence of the src-family kinases, Lck and Fyn, on T cell differentiation, survival and activation," Immunological Reviews, vol. 191, pp. 107-118, 2003.

[5] A. Veillette, I. D. Horak, E. M. Horak, M. A. Bookman, and J. B. Bolen, "Alterations of the lymphocyte-specific protein tyrosine kinase (p56(lck)) during T-cell activation," Molecular and Cellular Biology, vol. 8, no. 10, pp. 4353-4361, 1988.

[6] C. E. Rudd, J. M. Trevillyan, J. D. Dasgupta, L. L. Wong, and S. F. Schlossman, "The CD4 receptor is complexed in detergent lysates to a protein-tyrosine kinase ( $p$ 58) from human $\mathrm{T}$ lymphocytes," Proceedings of the National Academy of Sciences of the United States of America, vol. 85, no. 14, pp. 5190-5194, 1988.

[7] H. L. Ostergaard, D. A. Shackelford, T. R. Hurley et al., "Expression of CD45 alters phosphorylation of the lck-encoded tyrosine protein kinase in murine lymphoma T-cell lines," Proceedings of the National Academy of Sciences of the United States of America, vol. 86, no. 22, pp. 8959-8963, 1989.

[8] L. M. L. Chow, M. Fournel, D. Davidson, and A. Veillette, "Negative regulation of T-cell receptor signalling by tyrosine protein kinase p50csk," Nature, vol. 365, no. 6442, pp. 156-160, 1993.

[9] D. B. Straus and A. Weiss, "Genetic evidence for the involvement of the lck tyrosine kinase in signal transduction through the $\mathrm{T}$ cell antigen receptor," Cell, vol. 70, no. 4, pp. 585-593, 1992.

[10] W. S. F. Wong and K. P. Leong, "Tyrosine kinase inhibitors: a new approach for asthma," Biochimica et Biophysica ActaProteins and Proteomics, vol. 1697, no. 1-2, pp. 53-69, 2004.

[11] K. C. Lee, I. Ouwehand, A. L. Giannini, N. S. Thomas, N. J. Dibb, and M. J. Bijlmakers, "Lck is a key target of imatinib and dasatinib in T-cell activation," Leukemia, vol. 24, no. 4, pp. 896900, 2010

[12] K. De Keersmaecker, M. Porcu, L. Cox et al., "NUP214ABL1-mediated cell proliferation in T-cell acute lymphoblastic leukemia is dependent on the LCK kinase and various interacting proteins," Haematologica, vol. 99, no. 1, pp. 85-93, 2014.

[13] N. Isakov and B. Biesinger, "Lck protein tyrosine kinase is a key regulator of T-cell activation and a target for signal intervention by Herpesvirus saimiri and other viral gene products," European Journal of Biochemistry, vol. 267, no. 12, pp. 3413-3421, 2000.

[14] T. Morio, T. Chatila, and R. S. Geha, "HIV glycoprotein gp120 inhibits TCR-CD3-mediated activation of fyn and lck," International Immunology, vol. 9, no. 1, pp. 53-64, 1997.

[15] R. Swart, I. K. Ruf, J. Sample, and R. Longnecker, "Latent membrane protein 2A-mediated effects on the phosphatidylinositol 3-kinase/Akt pathway," Journal of Virology, vol. 74, no. 22, pp. 10838-10845, 2000.

[16] N.-H. Cho, P. Feng, S.-H. Lee et al., "Inhibition of T cell receptor signal transduction by tyrosine kinase-interacting protein of
Herpesvirus saimiri," Journal of Experimental Medicine, vol. 200, no. 5, pp. 681-687, 2004.

[17] L. V. Meléndez, R. D. Hunt, M. D. Daniel, B. J. Blake, and F. G. Garcia, "Acute lymphocytic leukemia in owl monkeys inoculated with Herpesvirus saimiri," Science, vol. 171, no. 3976, pp. 1161-1163, 1971.

[18] B. Biesinger, I. Muller-Fleckenstein, B. Simmer et al., "Stable growth transformation of human T lymphocytes by Herpesvirus saimiri," Proceedings of the National Academy of Sciences of the United States of America, vol. 89, no. 7, pp. 3116-3119, 1992.

[19] E. Meinl, B. A. 't Hart, R. E. Bontrop et al., "Activation of a myelin basic protein-specific human $\mathrm{T}$ cell clone by antigenpresenting cells from rhesus monkeys," International Immunology, vol. 7, no. 9, pp. 1489-1495, 1995.

[20] J.-P. Vernot, L. A. Pérez-Quintero, A. M. Perdomo-Arciniegas, S. Quijano, and M. E. Patarroyo, "Herpesvirus saimiri immortalization of Aotus T lymphocytes specific for an immunogenically modified peptide of Plasmodium falciparum merozoite surface antigen 2," Immunology \& Cell Biology, vol. 83, no. 1, pp. 67-74, 2005.

[21] S. M. Duboise, J. Guo, S. Czajak, R. C. Desrosiers, and J. U. Jung, "STP and Tip are essential for Herpesvirus saimiri oncogenicity," Journal of Virology, vol. 72, no. 2, pp. 1308-1313, 1998.

[22] L.-E. Wehner, N. Schröder, K. Kamino, U. Friedrich, B. Biesinger, and U. Rüther, "Herpesvirus saimiri Tip gene causes T-cell lymphomas in transgenic mice," DNA and Cell Biology, vol. 20, no. 2, pp. 81-88, 2001.

[23] J. Park, N.-H. Cho, J.-K. Choi, P. Feng, J. Choe, and J. U. Jung, "Distinct roles of cellular Lck and p80 proteins in herpesvirus saimiri Tip function on lipid rafts," Journal of Virology, vol. 77, no. 16, pp. 9041-9051, 2003.

[24] B. Biesinger, A. Y. Tsygankov, H. Fickenscher et al., "The product of the Herpesvirus saimiri open reading frame 1 (Tip) interacts with T cell-specific kinase p56lck in transformed cells," Journal of Biological Chemistry, vol. 270, no. 9, pp. 4729-4734, 1995.

[25] N. Wiese, A. Y. Tsygankov, U. Klauenberg, J. B. Bolen, B. Fleischer, and B. M. Bröker, "Selective activation of T cell kinase p56lck by Herpesvirus saimiri protein tip," Journal of Biological Chemistry, vol. 271, no. 2, pp. 847-852, 1996.

[26] P. Kjellen, K. Amdjadi, T. C. Lund, P. G. Medveczky, and B. M. Sefton, "The Herpesvirus saimiri Tip484 and Tip488 proteins both stimulate Lck tyrosine protein kinase activity in vivo and in vitro," Virology, vol. 297, no. 2, pp. 281-288, 2002.

[27] T. Lund, M. M. Medveczky, and P. G. Medveczky, "Herpesvirus saimiri tip-484 membrane protein markedly increases p56lck activity in T cells," Journal of Virology, vol. 71, no. 1, pp. 378-382, 1997.

[28] J. U. Jung, S. M. Lang, T. Jun, T. M. Roberts, A. Veillette, and R. C. Desrosiers, "Downregulation of Lck-mediated signal transduction by Tip of Herpesvirus saimiri," Journal of Virology, vol. 69, no. 12, pp. 7814-7822, 1995.

[29] J. Favero and V. Lafont, "Effector pathways regulating T cell activation," Biochemical Pharmacology, vol. 56, no. 12, pp. 15391547, 1998.

[30] J. U. Jung, S. M. Lang, U. Friedrich et al., "Identification of Lck-binding elements in Tip of Herpesvirus saimiri," Journal of Biological Chemistry, vol. 270, no. 35, pp. 20660-20667, 1995.

[31] T. C. Lund, P. C. Prator, M. M. Medveczky, and P. G. Medveczky, "The Lck binding domain of Herpesvirus saimiri Tip-484 constitutively activates Lck and STAT3 in T cells," Journal of Virology, vol. 73, no. 2, pp. 1689-1694, 1999. 
[32] D. A. Hartley, K. Amdjadi, T. R. Hurley, T. C. Lund, P. G. Medveczky, and B. M. Sefton, "Activation of the Lck tyrosine protein kinase by the Herpesvirus saimiri Tip protein involves two binding interactions," Virology, vol. 276, no. 2, pp. 339-348, 2000.

[33] E. Heck, U. Friedrich, M. U. Gack et al., "Growth transformation of human $\mathrm{T}$ cells by herpesvirus saimiri requires multiple TipLck interaction motifs," Journal of Virology, vol. 80, no. 20, pp. 9934-9942, 2006.

[34] S. M. Duboise, H. Lee, J. Guo et al., "Mutation of the Lckbinding motif of tip enhances lymphoid cell activation by Herpesvirus saimiri," Journal of Virology, vol. 72, no. 4, pp. 26072614, 1998.

[35] N.-H. Cho, D. Kingston, H. Chang et al., "Association of Herpesvirus saimiri Tip with lipid raft is essential for downregulation of T-cell receptor and CD4 coreceptor," Journal of Virology, vol. 80, no. 1, pp. 108-118, 2006.

[36] S. K. Bromley, W. R. Burack, K. G. Johnsonn et al., "The immunological synapse," Annual Review of Immunology, vol. 19, pp. 375-396, 2001.

[37] P. S. Kabouridis, "Lipid rafts in $\mathrm{T}$ cell receptor signalling (review)," Molecular Membrane Biology, vol. 23, no. 1, pp. 4957, 2006.

[38] J. J. Merlo and A. Y. Tsygankov, "Herpesvirus saimiri oncoproteins Tip and StpC synergistically stimulate NF- $\kappa$ B activity and interleukin-2 gene expression," Virology, vol. 279, no. 1, pp. 325338, 2001.

[39] D. Filipp, O. Ballek, and J. Manning, "Lck, membrane microdomains, and TCR triggering machinery: defining the new rules of engagement," Frontiers in Immunology, vol. 3, article 155, 2012.

[40] C.-K. Min, S.-Y. Bang, B.-A. Cho et al., "Role of amphipathic helix of a herpesviral protein in membrane deformation and T cell receptor downregulation," PLoS Pathogens, vol. 4, no. 11, Article ID e1000209, 2008.

[41] R. A. Houghten, "General method for the rapid solid-phase synthesis of large numbers of peptides: specificity of antigenantibody interaction at the level of individual amino acids," Proceedings of the National Academy of Sciences of the United States of America, vol. 82, no. 15, pp. 5131-5135, 1985.

[42] R. C. Judd, “Electrophoresis of peptides," Methods in Molecular Biology, vol. 32, pp. 49-57, 1994.

[43] A. Pinzoń-Charry, J. P. Vernot, R. Rodríguez, and M. E. Patarroyo, "Proliferative response of peripheral blood lymphocytes to mitogens in the owl monkey Aotus nancymae," Journal of Medical Primatology, vol. 32, no. 1, pp. 31-38, 2003.

[44] M. Croft and C. Dubey, "Accessory molecule and costimulation requirements for CD4 T cell response," Critical Reviews in Immunology, vol. 17, no. 1, pp. 89-118, 1997.

[45] G. J. J. C. Boonen, B. A. Van Oirschot, A. Van Diepen et al., "Cyclin D3 regulates proliferation and apoptosis of leukemic T cell lines," Journal of Biological Chemistry, vol. 274, no. 49, pp. 34676-34682, 1999.

[46] P. Otáhal, P. Angelisová, M. Hrdinka et al., "A new type of membrane raft-like microdomains and their possible involvement in TCR signaling," Journal of Immunology, vol. 184, no. 7, pp. 36893696, 2010.

[47] D. Filipp and M. Julius, "Lipid rafts: resolution of the 'fyn problem'?" Molecular Immunology, vol. 41, no. 6-7, pp. 645-656, 2004.
[48] D. Filipp, B. Moemeni, A. Ferzoco et al., "Lck-dependent Fyn activation requires $C$ terminus-dependent targeting of kinaseactive Lck to lipid rafts," The Journal of Biological Chemistry, vol. 283, no. 39, pp. 26409-26422, 2008.

[49] J. E. Ferrell Jr. and R. R. Bhatt, "Mechanistic studies of the dual phosphorylation of mitogen-activated protein kinase," The Journal of Biological Chemistry, vol. 272, no. 30, pp. 19008-19016, 1997.

[50] D.-W. Yoon, H. Lee, W. Seol, M. DeMaria, M. Rosenzweig, and J. U. Jung, "Tap: a novel cellular protein that interacts with tip of Herpesvirus saimiri and induces lymphocyte aggregation," Immunity, vol. 6, no. 5, pp. 571-582, 1997.

[51] A. G. Baxter and P. D. Hodgkin, "Activation rules: the two-signal theories of immune activation," Nature Reviews Immunology, vol. 2, no. 6, pp. 439-446, 2002.

[52] J. Guo, M. Duboise, H. Lee et al., "Enhanced downregulation of Lck-mediated signal transduction by a Y114 mutation of herpesvirus saimiri Tip," Journal of Virology, vol. 71, no. 9, pp. 7092-7096, 1997.

[53] S. C. Meuer, J. C. Hodgdon, R. E. Hussey, J. P. Protentis, S. F. Schlossman, and E. L. Reinherz, "Antigen-like effects of monoclonal antibodies directed at receptors on human $\mathrm{T}$ cell clones," Journal of Experimental Medicine, vol. 158, no. 3, pp. 988-993, 1983.

[54] J. E. Casnellie, "Sites of in vivo phosphorylation of the T cell tyrosine protein kinase in LSTRA cells and their alteration by tumor-promoting phorbol esters," Journal of Biological Chemistry, vol. 262, no. 20, pp. 9859-9864, 1987.

[55] J. D. Marth, D. B. Lewis, C. B. Wilson, M. E. Gearn, E. G. Krebs, and R. M. Perlmutter, "Regulation of pp56lck during Tcell activation: functional implications for the src-like protein tyrosine kinases," The EMBO Journal, vol. 6, no. 9, pp. 27272734, 1987.

[56] S. Choi and R. H. Schwartz, "Molecular mechanisms for adaptive tolerance and other T cell anergy models," Seminars in Immunology, vol. 19, no. 3, pp. 140-152, 2007.

[57] A. D. Holdorf, K.-H. Lee, W. R. Burack, P. M. Allen, and A. S. Shaw, "Regulation of Lck activity by CD4 and CD28 in the immunological synapse," Nature Immunology, vol. 3, no. 3, pp. 259-264, 2002.

[58] A. Veillette and M. Fournel, "The CD4 associated tyrosine protein kinase p56lck is positively regulated through its site of autophosphorylation," Oncogene, vol. 5, no. 10, pp. 1455-1462, 1990.

[59] M. E. M. Noble, J. A. Endicott, and L. N. Johnson, "Protein kinase inhibitors: insights into drug design from structure," Science, vol. 303, no. 5665, pp. 1800-1805, 2004.

[60] Z.-Y. Zhang, B. Zhou, and L. Xie, "Modulation of protein kinase signaling by protein phosphatases and inhibitors," Pharmacology \& Therapeutics, vol. 93, no. 2-3, pp. 307-317, 2002.

[61] R. J. He, Z. H. Yu, R. Y. Zhang, and Z. Y. Zhang, "Protein tyrosine phosphatases as potential therapeutic targets," Acta Pharmacologica Sinica, vol. 35, no. 10, pp. 1227-1246, 2014. 


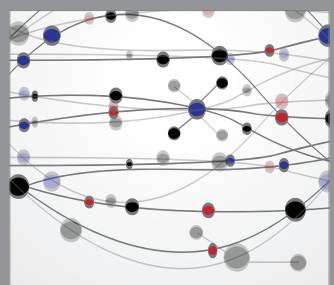

The Scientific World Journal
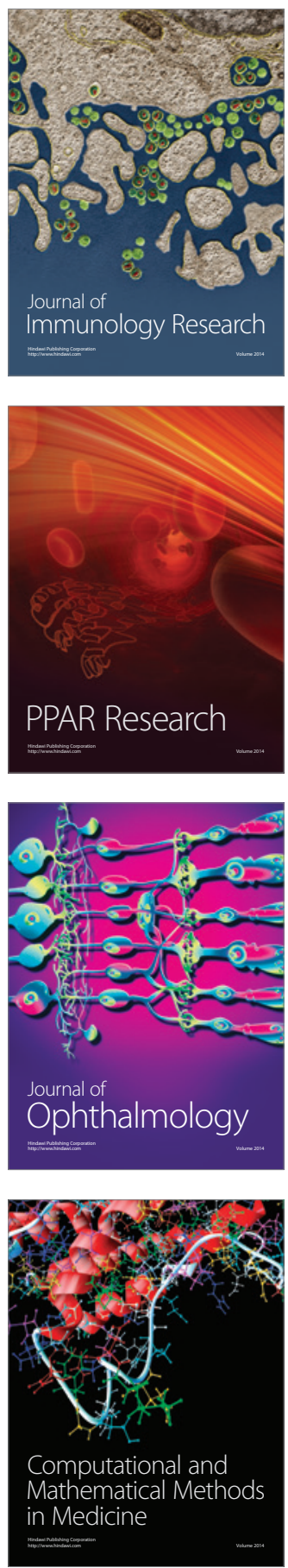

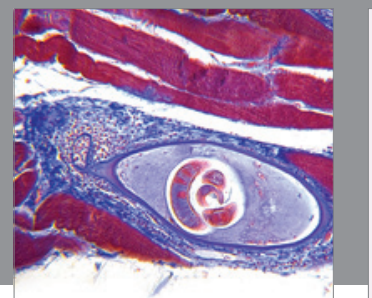

Gastroenterology

Research and Practice
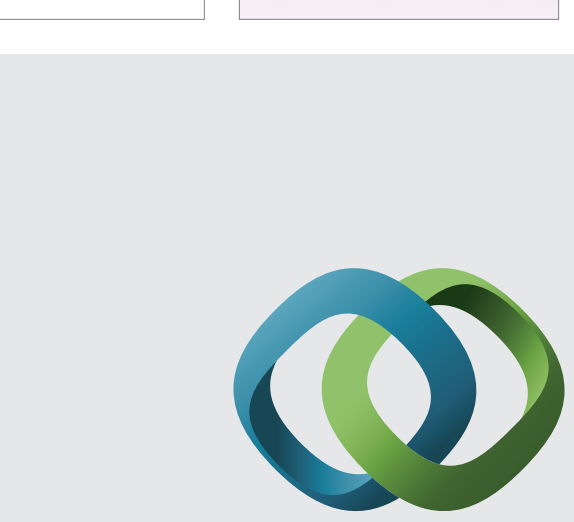

\section{Hindawi}

Submit your manuscripts at

http://www.hindawi.com
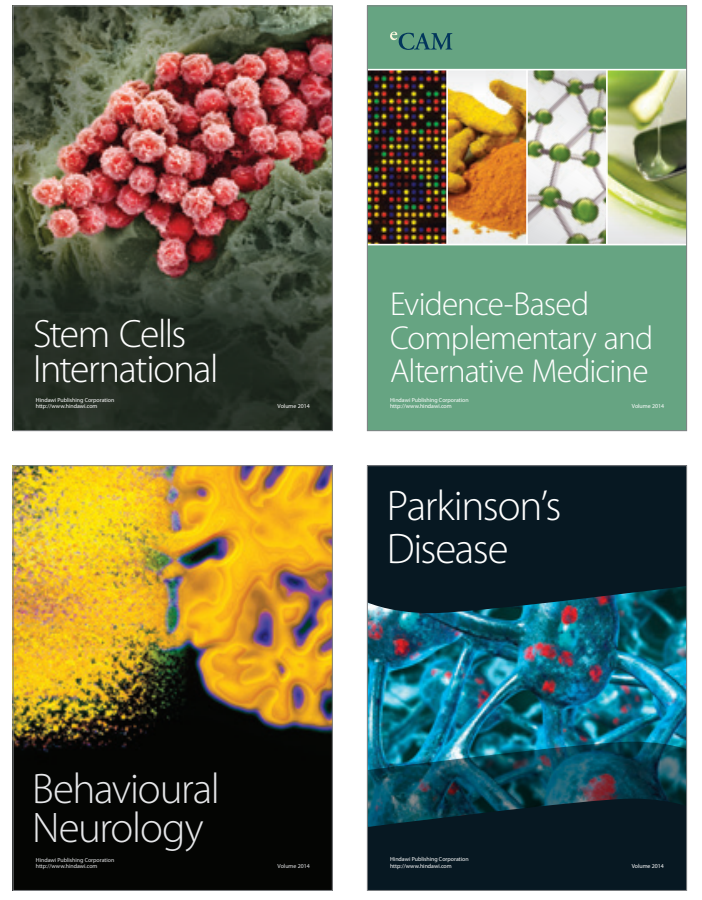
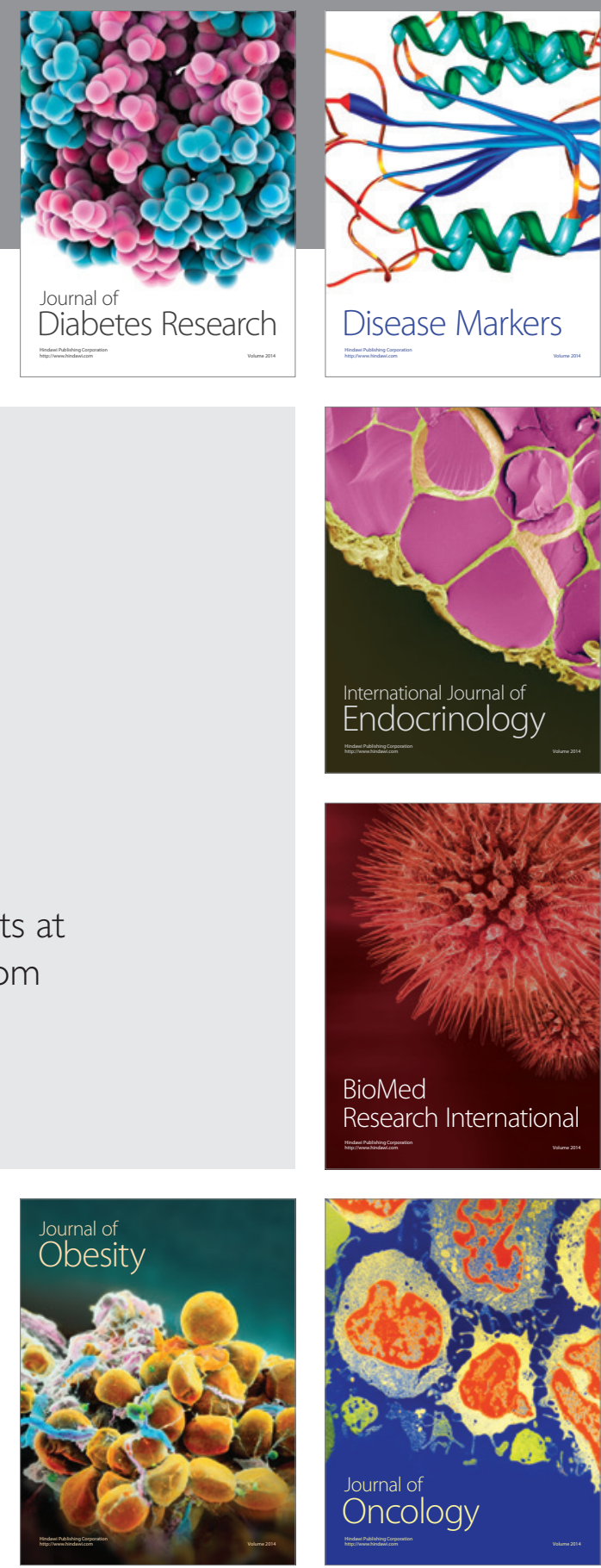

Disease Markers
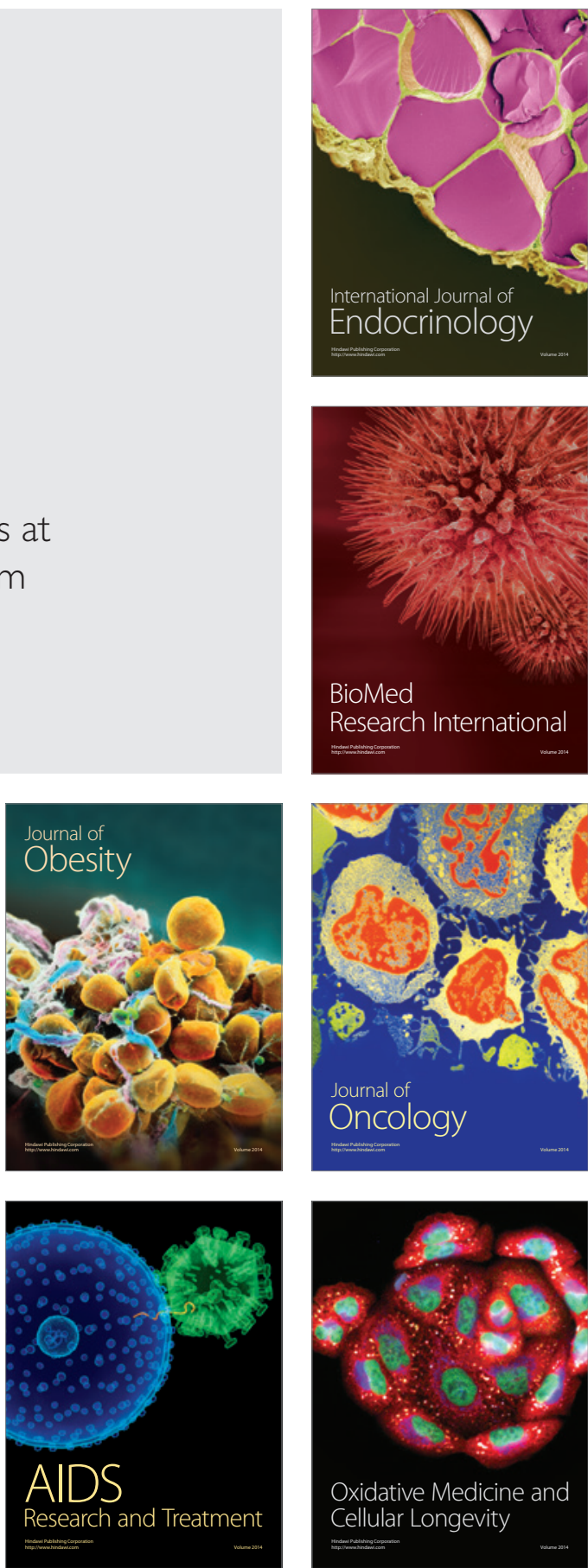